\title{
Nonlinear Controller Design of the Inverted Pendulum System based on Extended State Observer
}

\author{
Limin Du, Fucheng Cao \\ College of Electronic Information Engineering, Changchun University, Changchun, 130022, China
}

Keywords: inverted pendulum system, nonlinear controller, linear extended state observer, disturbance

\begin{abstract}
Inverted pendulum system is a kind of widely used physical model. In this paper, we present a nonlinear control scheme of single inverted pendulum system based on extended state observer. First, we establish the dynamic equations of inverted pendulum under a generic horizontal force and then linearize the inverted pendulum around the unstable upright position. Second, linear extended state observer is designed, which can observe the uncertain part of the system and various disturbances. Third, nonlinear feedback controller is designed so that the system has a stronger disturbance rejection capability. The experimental results show that the nonlinear control method based on extended state observer is effective. Because the designed control algorithm is simple, the parameters are easy to be tuning, and control effect is good, it has important reference value for similar control system with the inverted pendulum.
\end{abstract}

\section{Introduction}

The inverted pendulum is an experiment platform, which can verify the control method and theory. The simulation or physical control of inverted pendulum is a typical scheme to test a control theory or method. Its control methods have extensive use in military, aerospace, robotics and general industrial fields, such as balance control in robot walking, verticality control in rocket launching and attitude control in satellite flight etc.

The goal of controlling the inverted pendulum is that, when it initially begins to deviate from the vertical open-loop unstable position, artificial control makes the inverted pendulum in dynamic equilibrium. The structure of inverted pendulum has a lot of kinds, but no matter what kind of structure, the inverted pendulum is a high order, nonlinear, multi variable, strong coupling system, and many techniques already exist for its solution [1-8], for example, PID control, fuzzy control, adaptive control, LQR control, sliding mode variable structure control, and so on.

In this paper, a single linear inverted pendulum system as the research object, the nonlinear control scheme is designed based on extended state observer. Extended state observer can observe the uncertain part of the system and various disturbances so on. Nonlinear feedback controller has a stronger disturbance rejection capability. The simulation results show that this control method is very feasible and effective.

\section{Mathematical model of inverted pendulum}

The inverted pendulum system is composed of horizontal movement electrical car and the single inverted pendulum supported by it, as shown in Fig.1. The pendulum is constrained to move on the $\mathrm{X}$ axis, and the control action is based on the horizontal displacements of the electrical cart. The goal of controlling the inverted pendulum is to make it kept in this upright position when it starts with some nonzero angle off the vertical position because of external interference. 


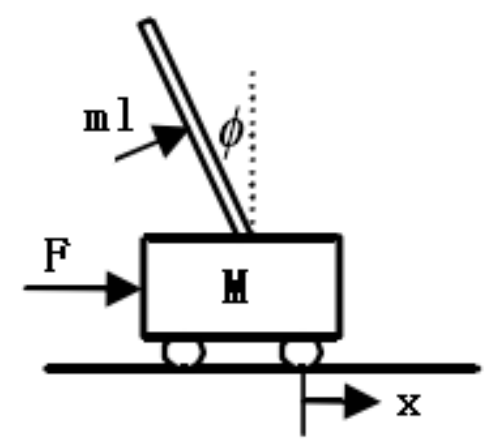

Fig.1. The mechanical model of single inverted pendulum

Where, $M$ is the quality of the cart, $m$ is the quality of the swing rod, $l$ is the length from pendulum mass to swing rod rotation axis, $F$ is the external force adding on the cart, $x$ is the horizontal displacement of the cart, $\phi$ is the angle between the swing rod and the vertical direction, $b$ is the friction coefficient between the cart and the ground.

By applying Lagrange's equations [9], we get the dynamic model of single inverted system:

$$
\left\{\begin{array}{l}
(M+m) \ddot{x}+b \dot{x}+m l \cos \phi \ddot{\phi}-m l \sin \phi \dot{\phi}^{2}=F \\
m l \cos \phi \ddot{x}+m l^{2} \ddot{\phi}-m g l \sin \phi=0
\end{array}\right.
$$

From (1), we can see that the inverted pendulum system is a nonlinear system. But we usually focus on the situation of practical interest of small inverted pendulum movements around the unstable open loop position. Therefore, we introduce the following approximations.

$$
\cos \phi=1, \quad \sin \phi=\phi, \quad \dot{\phi}^{2} \quad<0 .
$$

Hence, (1) can be transformed into (3)

$$
\left\{\begin{array}{l}
(M+m) \ddot{x}+b \dot{x}+m l \ddot{\phi}=F \\
\ddot{x}+l \ddot{\phi}-g \phi=0
\end{array}\right.
$$

(3)

For (3), the further simplification equation about $\phi$ is as follows:

$$
\ddot{\phi}=\frac{(M+m) g \phi}{M l}+\frac{b \dot{x}}{M l}-\frac{F}{M l}
$$

That is

$\ddot{\phi}=f(\phi)+\lambda F$

(5)

Where, $\quad f(\phi)=\frac{(M+m) g \phi}{M l}+\frac{b \dot{x}}{M l}, \quad \lambda=-\frac{1}{M l}$.

\section{Nonlinear control of inverted pendulum based on extended state observer}

\section{Design of extended state observer}

The extended state observer by using the method of double channel compensation changes the plant's mathematical model, which takes the nonlinear, uncertain, disturbance and dynamic coupling as an expansion state, then observes it and gives online compensation [10].

Let $x_{1}$ equal to $\phi, x_{2}$ equal to $\dot{\phi}, x_{3}$ equal to $f(\phi)$, then the extended state equation of (5) is designed as follows: 


$$
\left\{\begin{array}{l}
\dot{x}_{1}=x_{2} \\
\dot{x}_{2}=x_{3}+\lambda F \\
\dot{x}_{3}=f(\phi) \\
y_{1}=x_{1}
\end{array}\right.
$$

The mathematical model of linear extended state observer (6) is designed as follows:

$$
\left\{\begin{array}{l}
e_{1}=z_{1}-y_{1} \\
\dot{z}_{1}=z_{2}-\beta_{1} e_{1} \\
\dot{z}_{2}=z_{3}-\beta_{2} e_{1}+\lambda F \\
\dot{z}_{3}=-\beta_{3} e_{1}
\end{array}\right.
$$

Where, $z_{1}, z_{2}$ and $z_{3}$ are the observations of $x, x$ and $f(\phi)$ respectively. $\beta_{1}, \beta_{2}$ and $\beta_{3}$ are adjusted parameters.

The characteristic equation of (7) is $s^{3}+\beta_{1} s^{2}+\beta_{2} s+\beta_{3}$. In general, the characteristic equation form of stable good and good transition process is $(s+w)^{3}$, which is equal to $s^{3}+3 w s^{2}+3 w^{2} s+w^{3}$. Therefore, the parameter $\beta_{1}, \beta_{2}$ and $\beta_{3}$ can be configured to $3 w, 3 w^{2}$ and $w^{3}$. Here, $w$ can be tuned according to the system bandwidth.

Design of nonlinear controller.

Let $F$ equal to $F_{0}-\frac{z_{3}}{\lambda}$, if $z_{3}$ can better observe $f(\phi)$, then (5) can be rewritten as:

$$
\begin{aligned}
\ddot{\phi} & =f(\phi)+\lambda F \\
& =z_{3}+\lambda\left(F_{0}-\frac{z_{3}}{\lambda}\right) \\
& =\lambda F_{0}
\end{aligned}
$$

The nonlinear error feedback control law can be designed as follows:

$$
F_{0}=k_{1} f a l\left(\varepsilon_{1}, \alpha, \delta\right)+k_{2} f a l\left(\varepsilon_{2}, \alpha, \delta\right)
$$

Where, $f a l(e, \alpha, \delta)=\left\{\begin{array}{l}|e|^{\alpha} \operatorname{sign}(e),|e|>\delta \\ \frac{e}{\delta^{1-\alpha}}, \quad|e| \leq \delta \quad 0<\alpha<1, \delta>0 .\end{array} \quad \varepsilon_{1}=-z_{1}, \quad \varepsilon_{2}=-z_{2}, k_{1}\right.$ and $k_{2}$ are adjusted gain, $\delta$ is linear interval width.

The nonlinear function $f a l(e, \alpha, \delta)$ is proved to be very effective nonlinear gain function, small error corresponds to a high gain, high error corresponds to a small gain, when the error is small, the function can prevent a high gain to void high frequency flutter. A lot of simulation proves that, for the nonlinear controller will take "orders" to restrain disturbance [11,12], and the performance of the control system is basically unchanged when controller parameter changes in a wide range, the controller has strong robustness.

\section{Simulation experiment}

To verify and compare the control performance of the proposed controller, the inverted pendulum simulation system is built in Matlab/Simulink, which is shown in Fig.2.

The parameters of the inverted pendulum are given as follows: $l=0.5 \mathrm{~m}$, $g=9.8 \mathrm{~m} / \mathrm{s}^{2}, b=0.1 \mathrm{~N} / \mathrm{m} \cdot \mathrm{s}, M=2.2 \mathrm{~kg}, \mathrm{~m}=0.1 \mathrm{~kg}$. The initial state of the inverted pendulum are as follows: $x(0)=7, \dot{x}(0)=0, \phi(0)=0.1 \mathrm{rad}, \dot{\phi}(0)=0$.

The designed controller parameters are as follows : $k_{1}=10, k_{2}=6, \alpha=0.5$, $\delta=0.005, \beta_{1}=60, \beta_{2}=1200, \beta_{3}=8000$. 


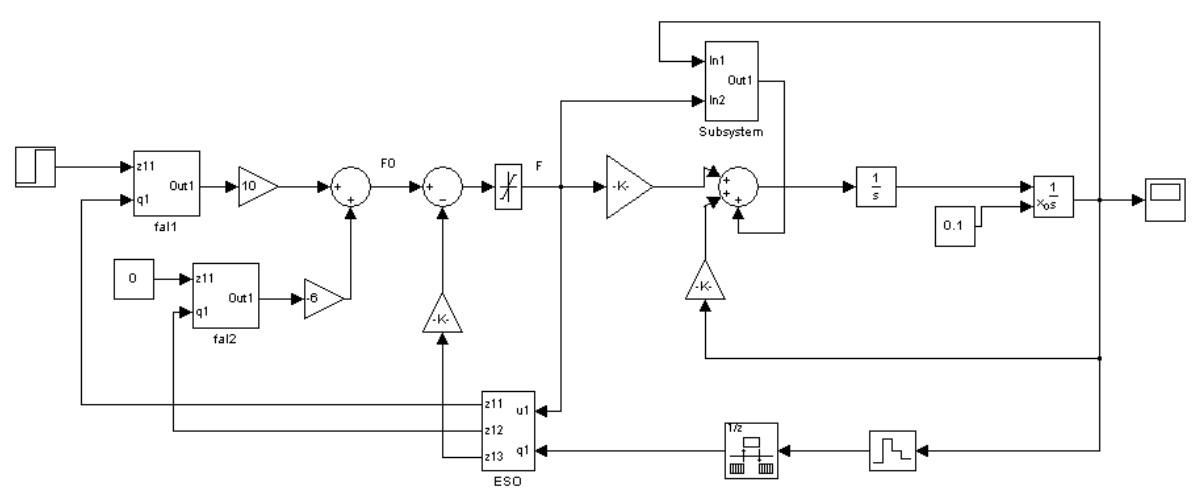

Fig.2. The simulation block diagram of the system

The speed sampling frequency is $200 \mathrm{~Hz}$. The control force $\mathrm{F}$ is limited to $10 \mathrm{~N}$. The simulation results of linear inverted pendulum stability control are shown as follows.

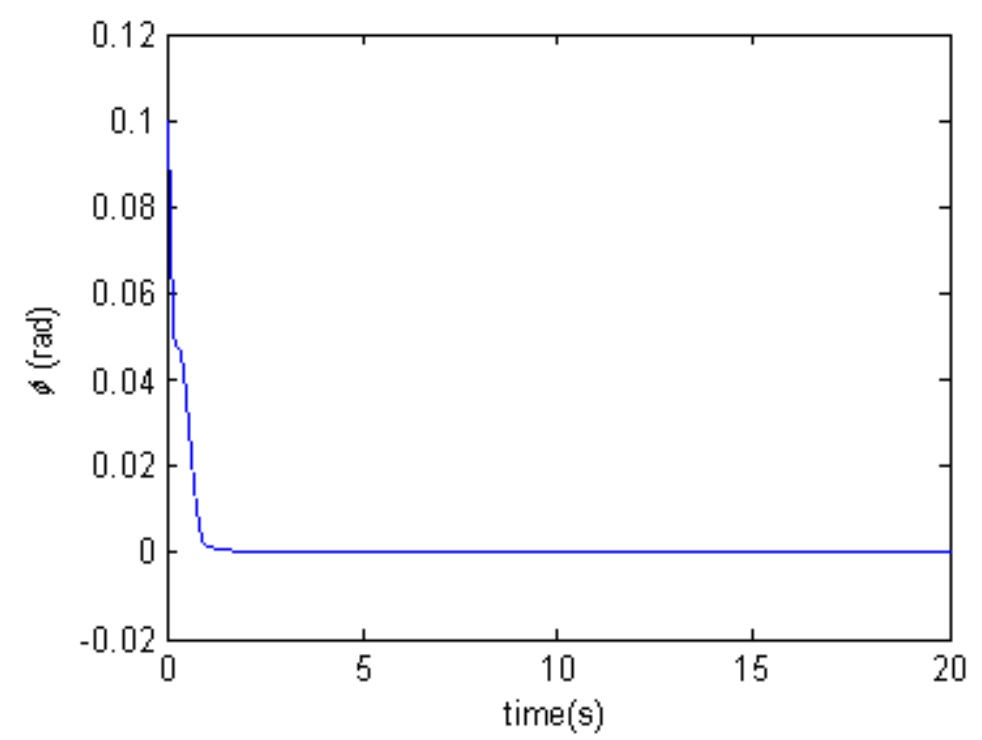

Fig.3. Angle response curve without external disturbance

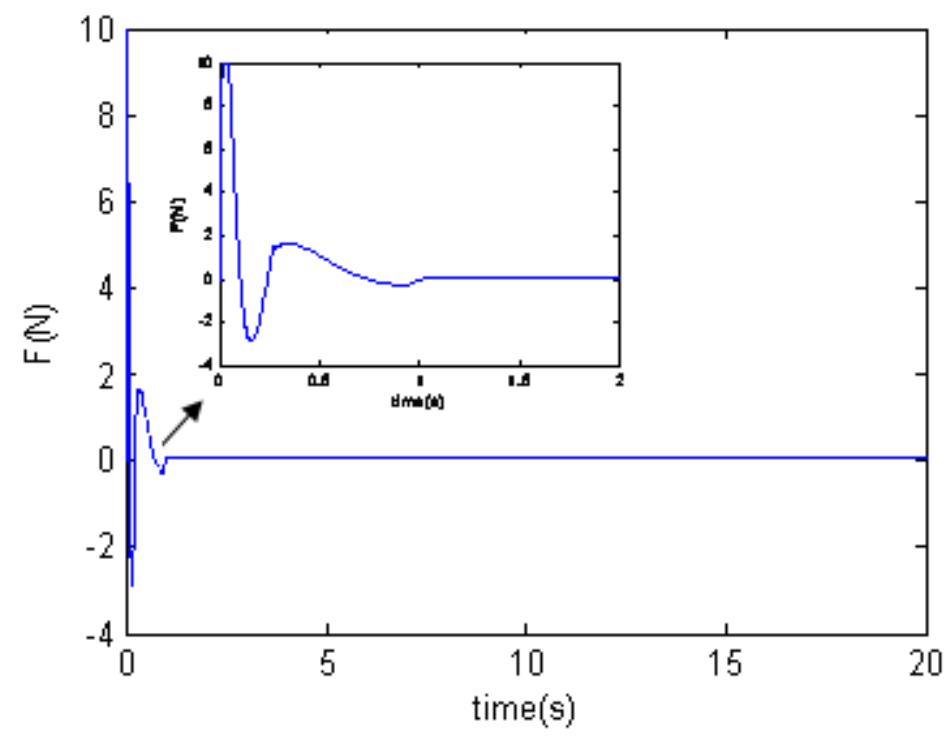

Fig.4. Control F curve without external disturbance

Fig. 3 shows that the designed linear inverted pendulum stable control system has good dynamic performance and steady state performance. Fig.4 is the curve of control F without external disturbance, which is restricted to $10 \mathrm{~N}$. 


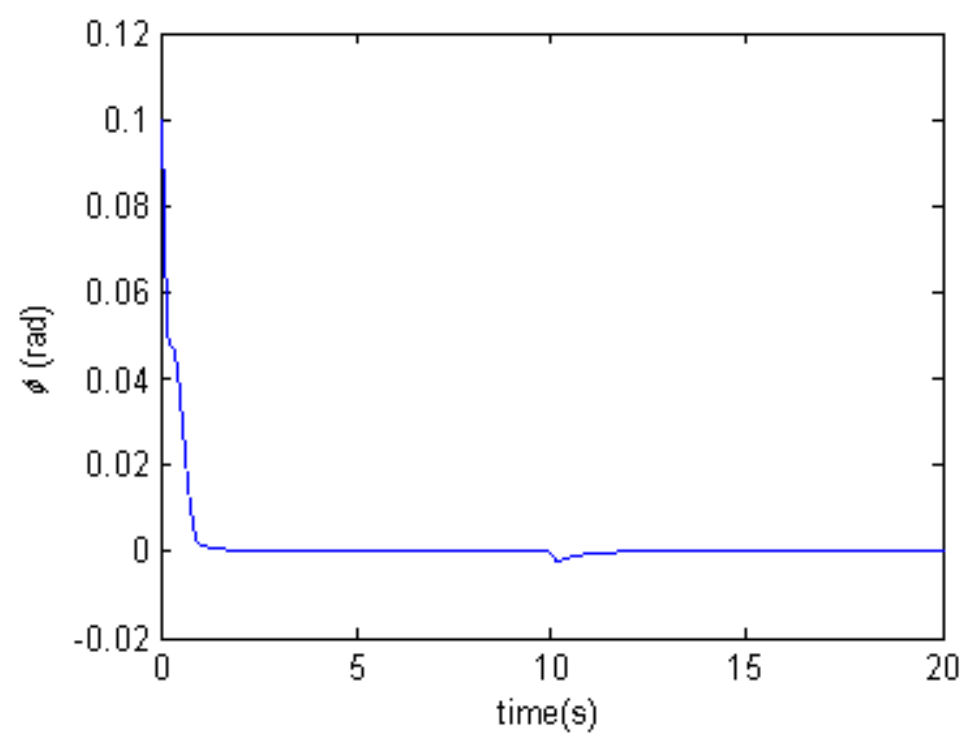

Fig.5. Angle response curve with external step disturbance

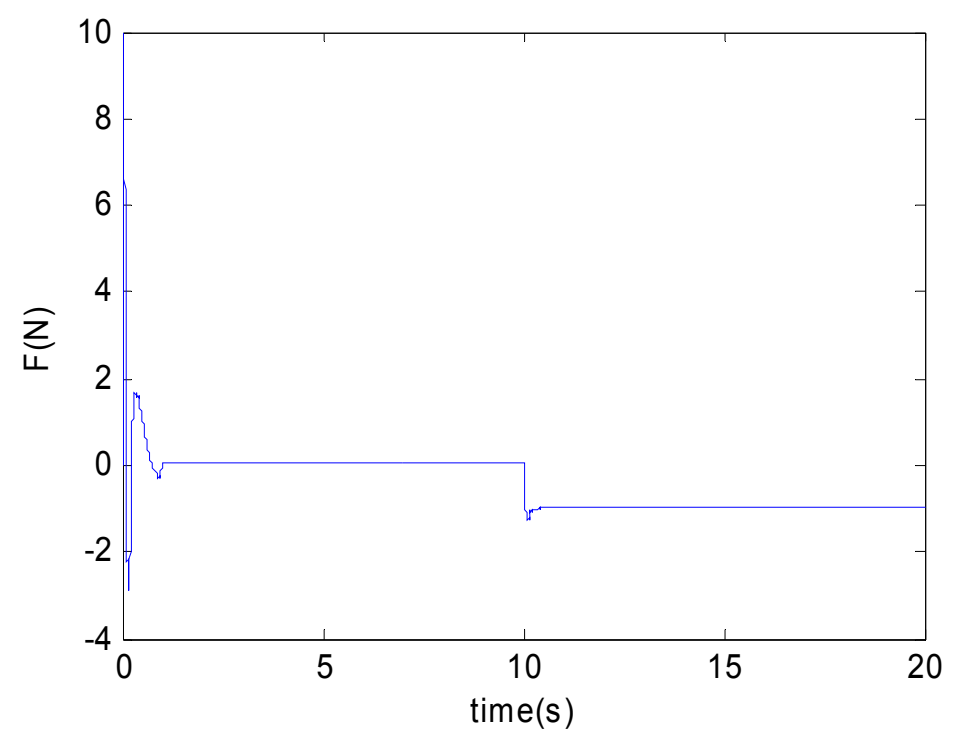

Fig.6. Control F curve with external step disturbance

In Fig. 5 and Fig.6, a step disturbance of $1 \mathrm{~N}$ is given in 10 second. Fig. 5 shows that the deviation angle of the swing rod increases when step disturbance is given, but the system is again back to the vertical stability state with 2 seconds, which achieves a better control effect. Fig. 6 is the curve of control F with step disturbance.

\section{Conclusions}

The inverted pendulum is a high order, nonlinear, multi variable, strong coupling system, and the real-time stability control of the inverted pendulum system in many areas has extensive use. This paper designs extended state observer to observe all sort of disturbances, and establishes a kind of nonlinear feedback controller. The simulation results show that the designed controller has better control performance and strong disturbance rejection capability, so it may have important reference value for similar control system with the inverted pendulum.

\section{References}

[1]WANG Shi-ying, ZHANG Feng, CHEN Zhi-yong. Design of a Linear Pendulum Optimal Controller Based on LQR. [J]. Machine Building \& Automation, 35 (6), pp. 95-98, 2006. 
[2] Li Jun, Yan Shi-liang. Design of Fuzzy-PID controller for first-order inverted pendulum. [J]. Foreign Electronic Measurement Technology, 31 (4), pp. 50-52, 2012.

[3] XIE Mu-jun, ZHAO Yan-hua, GAO Xing-hua. Application and Simulation Study of the Backstepping-sliding Mode-control on Pendulum. [J]. Control Engineering of China, 13, pp. 162-163, 2006.

[4] DUAN Xue-chao, QIU Yuan-ying, DUAN Bao-yan. Adaptive sliding mode fuzzy control of planar inverted pendulum .[J]. Control and Decision, 22 (7), pp. 774-777, 2007.

[5] WANG Guo-zheng, LI Hong, DONG Zong-hui. Stability Control of Planar Single Inverted Pendulum [J]. Industrial Control Computer , 26(3), pp.36-39, 2013.

[6] WANG Jia-Jun, LIU Dong-Liang, WANG Bao-Jun. Research on One Type of Saturated Nonlinear Stabilization Control Method of X-Z Inverted Pendulum [J]. Acta Automatica Sinica, 39(1), pp.92-96, 2013.

[7] Srinivasan B, Huguenin P, Bonvin D. Global stabilization of an inverted pendulum-control strategy and experimental verification. Automatica, 45(1), pp.265-269, 2009.

[8] Maravall D, Zhou C J, Alonso J. Hybrid fuzzy control of the inverted pendulum via vertical forces. International Journal of Intelligent Systems, 20(2), pp.195-211, 2005.

[9] Maravall D. Control and stabilization of the inverted pendulum via vertical forces. In:Tarn T-J, Chen SB, Zhou C, editors. Robotic welding, intelligence and automation. Lecture Notes in Control and Information Sciences 299. Berlin: Springer-Verlag; pp. 190-211, 2004.

[10]WANG Shuai, LI Hong-wen, MENG Hao-ran,WU. Active disturbance rejection controller for speed-loop in telescope servo system. [J]. Optics and Precision Engineering, China, 19 (10), pp. 2443-2448, 2011.

[11]YANG Sheng-jiang, GUO Jian-guo, GE Zhi-lei. Active disturbance rejection control application to interceptor attitude control system. [J]. FLIGHT DYNAMICS, 28 (3) , pp. 78-81, 2010.

[12] Li Jie, Qi Xiaohui, Han Shuaitao. Designing a New Nonlinear PID Attitude Controller for Small Quad-rotor Aircraft. [J]. Mechanical Science and Technology for Acrospace Engineering, 32 (8), pp. 1163-1166, 2013. 